ARTICLE

DOI: $10.1038 /$ ncomms13698 OPEN

\title{
Water striders adjust leg movement speed to optimize takeoff velocity for their morphology
}

Eunjin Yang ${ }^{1}$, Jae Hak Son ${ }^{2}$, Sang-im Lee ${ }^{3}$, Piotr G. Jablonski ${ }^{2,4} \&$ Ho-Young Kim ${ }^{1,5}$

Water striders are water-walking insects that can jump upwards from the water surface. Quick jumps allow striders to avoid sudden dangers such as predators' attacks, and therefore their jumping is expected to be shaped by natural selection for optimal performance. Related species with different morphological constraints could require different jumping mechanics to successfully avoid predation. Here we show that jumping striders tune their leg rotation speed to reach the maximum jumping speed that water surface allows. We find that the leg stroke speeds of water strider species with different leg morphologies correspond to mathematically calculated morphology-specific optima that maximize vertical takeoff velocity by fully exploiting the capillary force of water. These results improve the understanding of correlated evolution between morphology and leg movements in small jumping insects, and provide a theoretical basis to develop biomimetic technology in semi-aquatic environments.

\footnotetext{
${ }^{1}$ Department of Mechanical and Aerospace Engineering, Seoul National University, Seoul 08826, Korea. ${ }^{2}$ Laboratory of Behavioral Ecology and Evolution, School of Biological Sciences, Seoul National University, Seoul 08826, Korea. ${ }^{3}$ Daegu-Gyeongbuk Institute of Science and Technology School of Undergraduate Studies, Daegu 42988, Korea. ${ }^{4}$ Museum and Institute of Zoology, Polish Academy of Sciences, Wilcza 64, Warszawa 00-679, Poland.

${ }^{5}$ Big Data Institute, Seoul National University, Seoul 08826, Korea. Correspondence and requests for materials should be addressed to P.G.J.

(email: piotrjab@behecolpiotrsangim.org) or to H.-Y.K. (email: hyk@snu.ac.kr).
} 
$t$ is widely known that the superhydrophobic hairy legs of water striders enable them to float only on $\operatorname{tarsi}{ }^{1,2}$, and that the striders transport the momentum via vortices and capillary waves to propel themselves across the water surface ${ }^{1,3-5}$. But what are the mechanical characteristics of jumping off the water surface? Vertical and near-vertical jumps are performed in natural habitats in a series of frequent jumps that are triggered by attacks of predators, such as fish and backswimmers (predatory insects of the genus Notonecta), from under the water surface ${ }^{6,7}$. While it was suggested that water striders push the water surface with their legs to generate the upward capillary force on jumping ${ }^{3,8-10}$, the mechanics of this swift mode of locomotion have not been fully understood. Recently, a robotic water strider was built that uses the basic principle of momentum transfer observed in one of the larger species of water striders, Aquarius paludum ${ }^{11}$. A simple mechanical model of interactions between $A$. paludum legs and the water surface has been created to aid designing the robot. But it is still uncertain whether the combinations of species-specific morphology and leg movements observed in water striders maximize the insects' jumping performance as expected from natural selection for predation avoidance. Upon the basis of the initial kinematic calculations ${ }^{11}$ and the insights from high speed videos of jumping, we develop here a theoretical model of insect leg movements that enables the predictions of conditions for optimal jump performance in water striders. We then empirically verify the optimal predictions using individuals from five water strider species of different body sizes and leg morphologies. We show that, despite having different morphological constraints on leg dimensions, species tune their leg rotation speed to optimize the takeoff velocity from the water surface.

\section{Results}

General description of a water strider's jumping on water. From high-speed videos (see Methods for details) of three species of water striders (Gerris latiabdominis, G. gracilicornis and A. paludum), we observed near vertical jumps (with trajectories steeper than $60^{\circ}$ to the horizontal; Supplementary Video 1) in order to develop the mathematical model of the vertical component of jumping. At rest, a water strider (Fig. 1a) supports its weight using all of six legs with its body centre located at $y_{i}$ (see list of symbols and their descriptions in Table 1) above the water surface. Once the insect initiates a jump by pushing the surface with the middle and hind legs downwards, dimples are made (Fig. 1a), which enables the insect to control its direction and speed of the jump by transferring momentum to the water. The dimple depth $h$ (Fig. 1b), measured from the unperturbed water surface, increases and then decreases with time $t$ (Fig. 1f). The dimple depth reaches its maximum $h_{m}$ at the time $t_{m}$, which divides the jump into two stages: the pushing $\left(t<t_{m}\right)$ and the closing $\left(t>t_{m}\right)$ stage. The average downward velocity of four legs with respect to horizontal plane through insect's body centre $v_{s}$ is bell-shaped over time (Fig. 1g), and the upward velocity of the insect body $v$ overtakes $v_{s}$ at the moment of reaching the maximum dimple depth, $t=t_{m}$, when the vertical growth rate of the dimple $\left(v_{s}-v\right)$ becomes zero and its corresponding maximal depth is denoted as $h_{m}$. Hence, in the pushing stage the downward velocity of legs is larger than the upward velocity of the insect body $\left(v_{s}>v\right)$, but in the closing stage $v_{s}<v$.

In the pushing stage, the tarsus and tibia of each leg (Fig. 1c) remain in contact with the water surface until $t_{m}\left(t_{m} \approx 13 \mathrm{~ms}\right.$ in Fig. 1e). Thus, the average wetted length of the legs $l_{w}$ is assumed to be almost constant and equal to the average of sum of tarsi and tibiae lengths of four legs $l_{t}$. In the closing stage $\left(t>t_{m}\right)$, the legs continue to come close together and slide on the water surface towards the body while gradually disengaging themselves from the water surface causing a decrease in $l_{w}$. Owing to the decreasing wetted length $l_{w}$ that interacts with the water surface, the increase rate in upward velocity of the insect body in the closing stage is lower than that in the pushing stage as shown in Fig. 1g.

Based on these observations, we build a model to calculate the vertical force that produces the vertical jump as a function of the depth of the dimple formed by the legs. The force can be used to estimate the takeoff velocity, which allows us to seek the optimal stroke condition that the water strider should perform to maximize jumping speed. Fast jumping is important for the water striders to escape from the predators, such as backswimmers or fish, attacking from under the water surface. Therefore, evolution of abilities to maximize takeoff speed is expected under natural selection.

Theoretical model. When a water strider strokes the water surface, forces of various origins are exerted on the insect's legs in addition to the capillary force $\left(F_{s} \sim \sigma l_{w}\right)$, including pressure force $\left(F_{p} \sim \rho U^{2} r l_{w}\right)$, buoyancy $\left(F_{b} \sim \rho g r h l_{w}\right)$, inertial force due to added mass $\left(F_{a} \sim \rho r^{2} l_{w} U^{2} / h\right)$, viscous force $\left(F_{v} \sim \mu r l_{w} U / l_{c}\right)$ and the weight of the water strider $\left(F_{w} \sim m g\right)$. Here $\sigma$ is surface tension coefficient, $\rho$ is density of liquid, $U$ rate of vertical growth of dimple, $r$ radius of the leg, $g$ gravitational acceleration, $\mu$ viscosity of liquid and $l_{c}=[\sigma /(\rho g)]^{1 / 2}$ capillary length of water. Our theoretical analysis used the standard values, such as density of water, $\rho=998 \mathrm{~kg} \mathrm{~m}^{-3}$; surface tension coefficient of water, $\sigma=0.072 \mathrm{~N} \mathrm{~m}^{-1}$; viscosity of water, $\mu=10^{-3} \mathrm{~Pa} \cdot s$; gravitational acceleration, $g=9.8 \mathrm{~m} \mathrm{~s}^{-2}$ and average values of experimentally measured parameters for jumping G. gracilicornis, the mediumsized water strider species: wetted length, $l_{w}=7.5 \mathrm{~mm}$ (evaluated from the average length of four pairs of tibia and tarsus); leg radius, $r=50 \mu \mathrm{m}$ (measured at the middle of the tibia); body mass of water strider, $m=30 \mathrm{mg}$; representative leg descending speed, $U=0.15 \mathrm{~m} \mathrm{~s}^{-1}$; and depth of dimple, $h=3 \mathrm{~mm}$. The ratios of the other forces to the capillary force are scaled as $F_{p} /$ $F_{s} \sim 10^{-2}, F_{b} / F_{s} \sim 10^{-2}, F_{a} / F_{s} \sim 10^{-4}, F_{v} / F_{s} \sim 10^{-5}$ and $F_{w} /$ $F_{s} \sim 10^{-1}$. These ratios suggest that the capillary force dominates over the other forces. Moreover, the ratio of energy loss $E_{d}$ when the leg becomes detached from the water surface to kinetic energy of the water strider taking off the surface, $E_{k} \sim m v^{2}$, are scaled as $E_{d} / E_{k} \sim 10^{-4}$, implying negligible energy loss due to wet adhesion. Here, the reported value of energy loss, $E_{d}$, of a leg of a water strider via detachment from the water surface is of the order of $10^{-9} \mathrm{~J}^{12}$, and $v \sim 1 \mathrm{~ms}^{-1}$.

Hence, the seemingly complex phenomenon of jumping on the water surface can be simplified as a surface tension-dominant interaction of a long thin flexible cylinder with the water surface. Assuming that all the legs involved in the propulsion move synchronously and leave the surface at the same time (Supplementary Fig. 1 and Supplementary Note 1), the upward force $F$ on four legs, which is equivalent to the weight of water displaced by the legs (creating the dimples), is estimated by modifying Vella's model ${ }^{13}$ (Supplementary Note 2)

$$
F=8 \rho g l_{c} C l_{w} h\left\{1-\left[h /\left(2 l_{c}\right)\right]^{2}\right\}^{1 / 2}
$$

where $C$ is the flexibility factor depending on the scaled leg length $L_{f}=l_{w} / l_{e c}$, and $l_{e c}=\left(B l_{c} / \sigma\right)^{1 / 4}$ being the modified elastocapillary length of the leg with the bending rigidity $B=\pi E r^{4} / 4, E$ being Young's modulus of insect cuticle and $r$ being radius of the leg. We approximated $C$ as $C \approx\left(1+0.082 L_{f}^{3.3}\right)^{-1}$ for $L_{f}<2$ and $C l_{w}$ could indicate the effective wetted leg length (Supplementary Fig. 2 and Supplementary Note 2). The force $F$ increases 

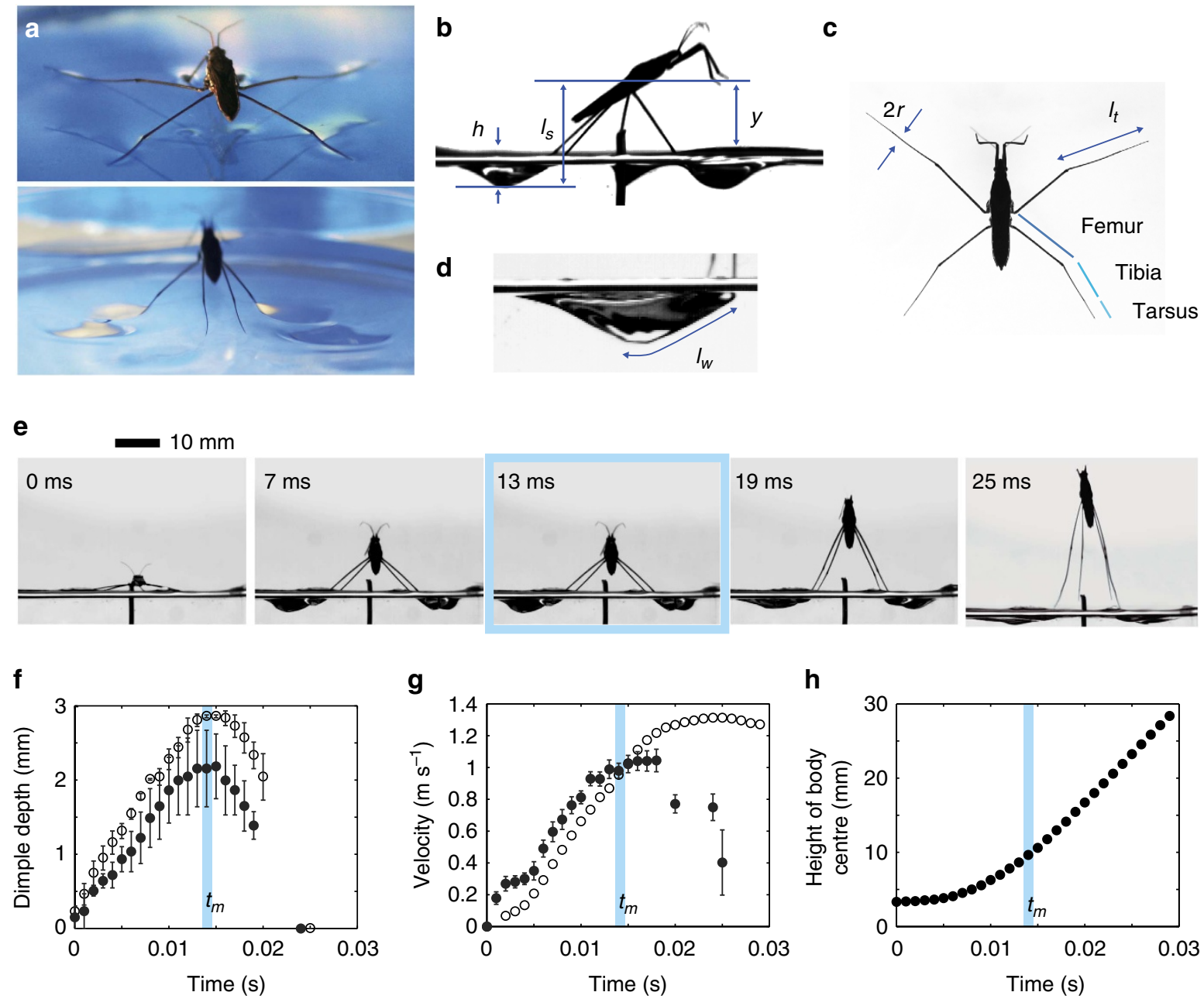

Figure 1 | Jumping of a water strider. (a) A water strider (male Aquarius paludum with a body mass of $37.2 \mathrm{mg}$ and an average length of middle and hind legs of $22.1 \mathrm{~mm}$ ) that rests and jumps on water. (b-d) Definitions of various lengths considered in this study. (b) The vertical lengths including body centre location $y$, vertical distance from the tip of the legs to the horizontal plane through body centre $I_{s}$ and dimple depth $h$. (c) The lengths of legs including the radius $r$, and the length of tibia plus tarsus $I_{t}$. $(\mathbf{d})$ The wetted length of the leg $I_{w}$. (e) A representative sequence of the jump of the water strider on the water surface. (f-h) Measurement data extracted from a movie corresponding to e. (f) Average dimple depth formed by the right and left legs (open circles for middle legs, filled circles for hind legs) during the jump. Error bars indicate standard deviation between right and left legs. (g) Vertical velocity of the body centre $v$ (open circles) and the average downward velocity of the four legs with respect to the horizontal plane through the body centre $v_{s}$ (filled circles). Error bars indicate standard deviation among four legs. (h) Height of the body centre of the water strider during the jump. The vertical blue bar in f-h indicates the moment when the dimple reaches the maximal depth (the panel corresponding to $13 \mathrm{~ms}$ in $\mathbf{e}$ shows the dimple that reached maximal depth). Body velocity profile in $\mathbf{g}$ is the same data as that of water strider 2 in Koh et al. ${ }^{11}$

monotonically with the depth of dimple $h$ until the surface penetration occurs.

From momentum conservation, the upward velocity of the centre of mass of the insect can be determined as $v=\int F \mathrm{~d} t / m$, with $m$ being the insect's body mass. Then, the temporal change of the dimple depth $h(t)$ is given by

$$
\frac{\mathrm{d} h}{\mathrm{~d} t}=v_{s}-\frac{1}{m} \int F \mathrm{~d} t .
$$

We approximated the relationship between the downward linear velocity of the legs $v_{s}$ and the angular velocity of leg rotation $\omega$ as a sinusoidal function: $v_{s}=\omega \Delta l \sin (2 \omega t)$, based on the vertical distance of the legs $l_{s}$, which was measured between the tip of the legs and the horizontal plane through the body centre and modelled as $l_{s}=\Delta l[1-1 / 2 \cos (2 \omega t)]+y_{i}$ during the stroke. Here the angular velocity of the leg rotation $\omega$ is assumed to not change during a jump. The angular velocity of leg rotation $\omega$ and the maximal (theoretical) downward reach of the legs
$\Delta l=l_{l}-y_{i}$, where $l_{l}$ is the average length of the four legs, are the parameters reflecting behavioural and morphological traits of each insect, respectively. This is one of the simplest models satisfying necessary conditions to imitate water striders' leg movements with respect to the body centre during the jump: the vertical distance of the legs $l_{s}$ increases from $y_{i}$ to $l_{l}$; while the downward linear velocity of the legs $v_{s}$ increases from zero and then decreases to zero with its maximum in the middle. We confirmed that this theoretical model matches well the movements of the real water striders' legs (Fig. 2a,b).

In the model, the angular velocity of leg rotation $\omega$ does not change during a jump. This concept is unrealistic, but to illustrate how close our theoretical approximation of leg rotation $\omega$ is to the real angular leg movements by jumping insects, we compared the time derivative of average angle of four legs with respect to the horizontal plane through the body centre, $\theta$ (extracted from the video), with the value of $\omega$ that leads to a good match between empirical and modelled vertical distance of the legs $l_{s}$ (Fig. 2a) 


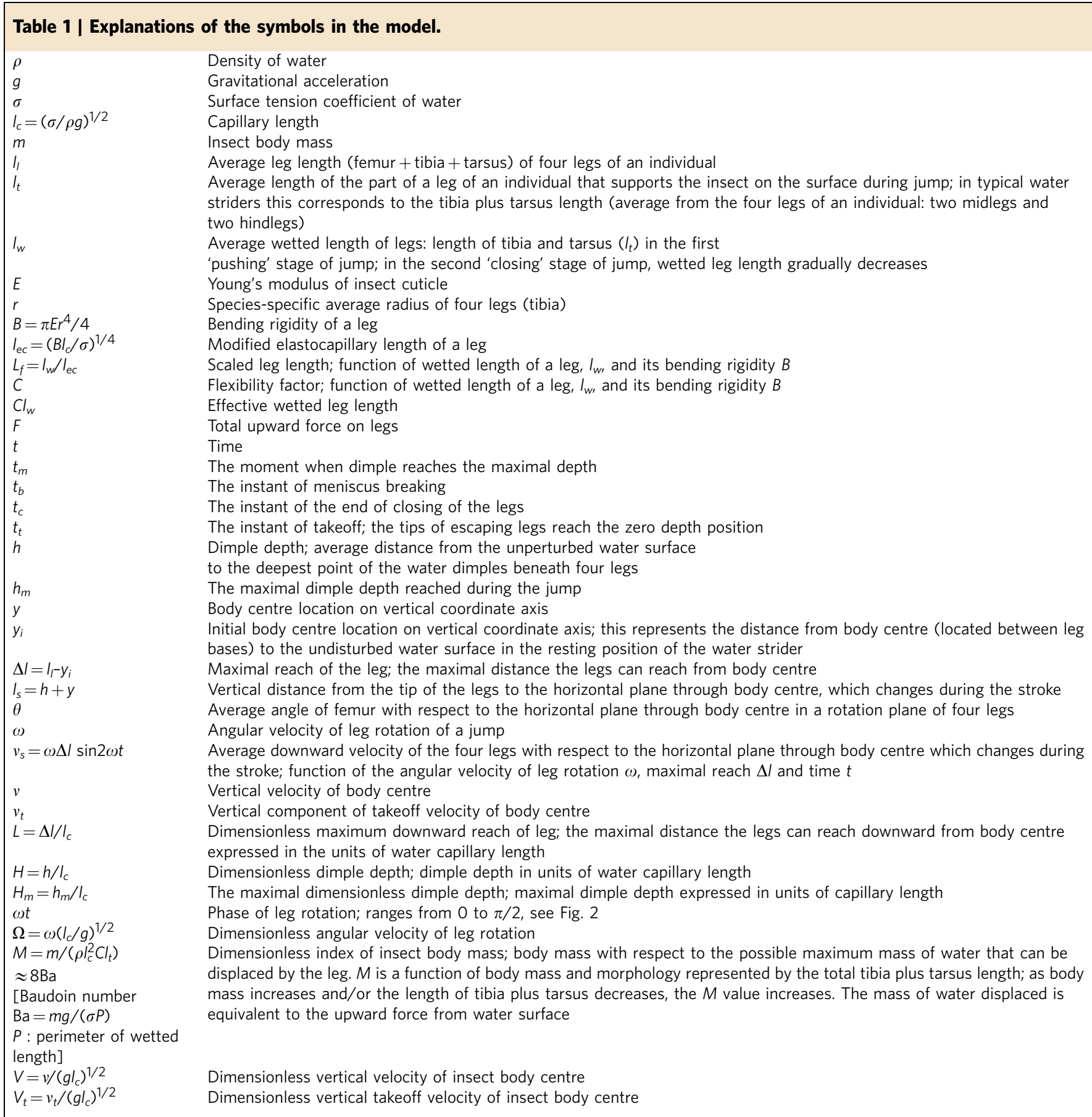

and downward linear velocity of the legs $v_{s}$ (Fig. 2b). We calculated the value of $\omega$ for each jump $\left(\omega=v_{s, \max } / \Delta l\right)$ with empirically measured $v_{s, \max }$ (maximum value of $v_{s}$ ) and $\Delta l$. We also calculated the time derivative of the empirically measured (from video) average angle of legs with respect to the horizontal plane through the body centre (Supplementary Fig. 3 and Supplementary Note 3 ). The value of $\dot{\theta}$ was not constant during the jump, but rather tended to quickly increase during the initial $8-10 \mathrm{~ms}$ of a jump, and then it fluctuated randomly (partly due to measurement error) indicating the apparent plateau as shown in Fig. 2c. Interestingly, the angle $\theta$ varies over time in a manner resulting in the changes of the vertical distance from the tip of the legs to the horizontal plane through body centre, $l_{s}$, approximating a sinusoidal function of $\omega t$. Note that
$l_{s}=\Delta l \sin \theta(t) \approx \Delta l[1-1 / 2 \cos (2 \omega t)]+y_{i}$. Furthermore, the value of $\omega$ is similar to the time average of empirical value of the average angular velocity of the leg rotation $\theta$ as seen in Fig. $2 c$. Therefore, we believe that our theoretical approach by using $\omega$ is a reasonable theoretical representation of the average angular velocity of leg rotation of vertically jumping water striders.

Combining equations (1) and (2), with the sinusoidal model of $v_{s}$, leads to a simple differential equation of the scaled dimple depth $H(\omega t)$ as

$$
\frac{\mathrm{d}^{2} H}{\mathrm{~d}(\omega t)^{2}}+\frac{8}{\Omega^{2} M} H\left(1-H^{2} / 4\right)^{1 / 2}-2 L \cos (2 \omega t)=0,
$$

where $H=h / l_{c}$ (dimensionless dimple depth), $\Omega=\omega\left(l_{c} / g\right)^{1 / 2}$ 

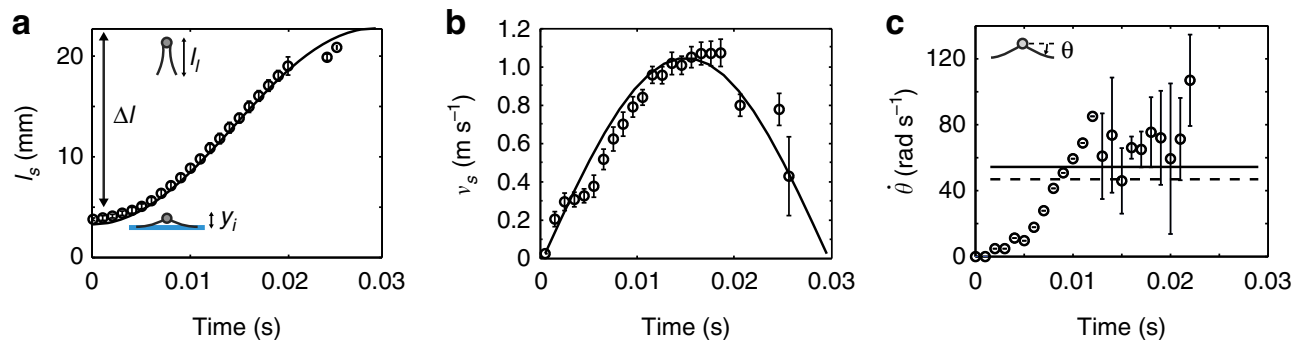

Figure 2 | Comparison of empirical and modelled leg movements. The solid lines correspond to the model assuming the sinusoidal model of leg rotation and the circles correspond to the average of measurement of four legs from the same movie used in Fig. 1e-h. The error bars indicate standard deviation among the four legs. (a) The average vertical distance between the body centre and distal end of legs $\left(I_{s}\right)$ across the leg rotation cycle. (b) The average downward velocity of four legs with respect to body centre $\left(v_{s}\right)$. (c) The average time derivative of the angle of legs with respect to the horizontal plane $(\dot{\theta})$. The dashed line indicates the time average of the measured values of $\dot{\theta}$ through the whole cycle and the solid line refers to the corresponding angular speed of leg rotation $\omega$ used in the model calculations of $I_{s}$ and $v_{s}$ in $\mathbf{a}, \mathbf{b}$.

a Pre-takeoff closing
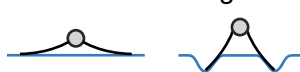

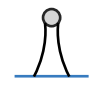

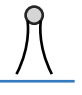

Post-takeoff closing
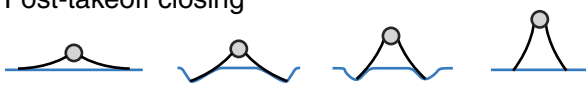

9

Meniscus breaking
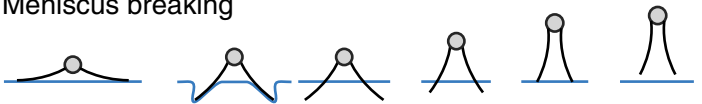

b
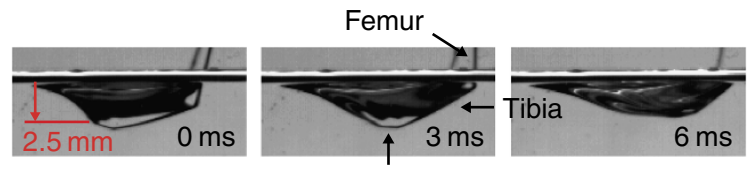

Tarsus

C

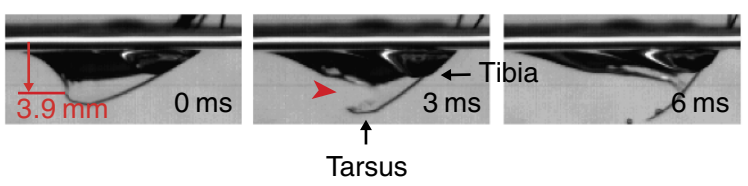

Figure 3 | Different jump modes. (a) Schematic representation of three modes of jump: pre-takeoff closing, post-takeoff closing and meniscus breaking jumps. (b,c) Enlarged images of the leg and dimple in a post-takeoff closing jump $\left(h_{m}=2.5 \mathrm{~mm}\right)$ and a meniscus breaking jump $\left(h_{m}>3.9 \mathrm{~mm}\right):(\mathbf{b})$ the leg that does not reach the sinking depth leaves the surface unpenetrated, (c) the leg pierces the surface just below the sinking depth. The red arrow at 3 ms indicates the rupture point of the water surface. The pre-takeoff closing jump was not observed in the experiments.

(dimensionless angular velocity of leg rotation), $M=m /\left(\rho l_{c}^{2} C l_{t}\right)$ (dimensionless index of insect body mass with respect to the maximum mass of water that can be displaced by the leg of the total tibia plus tarsus length $l_{t}$, which is directly related to the maximum supporting force of the water surface), and $L=\Delta l / l_{c}$ (dimensionless maximum downward reach of leg). $M \approx 8 \mathrm{Ba}$ where Baudoin number $\mathrm{Ba}=m g /(\sigma P)=1$, with $P$ the perimeter of wetted parts of legs, implies the maximum body weight that capillary force can support. Here, $\omega t$ is the phase of leg rotation, being 0 at the beginning and $\pi / 2$ at the end of the stroke (the range $[0-\pi / 2]$ is due to the sinusoidal approximation of the leg movements).

In the pushing stage $\left(v_{s}>v\right)$, the observed visible wetted legs $l_{w}$ comprised tibia and tarsi, $l_{t}$. Therefore, the wetted length in the pushing stage is estimated to be $l_{w} \approx l_{t}$, leading to $M \approx m /\left(\rho l_{c}^{2} C l_{t}\right)$. However, in the closing stage $\left(v_{s}<v\right)$, the wetted leg length $l_{w}$ decreases gradually while the legs disengage themselves from the water (Supplementary Video 1). In addition, as the legs close in the closing stage their inclination angles from the water surface increase leading to an apparent decrease of flexibility factor $C^{13}$.

To consider these changes in $l_{w}$ and $C$, while solving the differential equation (3) of dimple growth and decay, we simplified the wetted length in the closing stage to $l_{w}=l_{t}(\pi / 2-\omega t) /\left(\pi / 2-\omega t_{m}\right)\left(H / H_{m}\right)$. This equation consists of three terms: the wetted length in pushing stage $l_{t}$, and the two terms that decrease with the increase of $\omega t$ and the corresponding decrease of $H$ respectively. Then, the temporal change of the dimple depth can be obtained by solving the differential equation with two pairs of initial conditions: $H(0) \approx 0$ corresponding to negligible initial dimple depth formed by the weight of the insect, and $H^{\prime}(0)=0$ at the beginning of the pushing stage; $H\left(\omega t_{m}\right)=H_{m}$ and $H^{\prime}\left(\omega t_{m}\right)=0$ at the instant of maximum dimple depth when the closing stage starts. The takeoff velocity of the water strider $v_{t}$ is defined as the velocity at the moment when the end tips of escaping legs reach zero depth position $\left(t=t_{t}\right.$, and $\left.H\left(t_{t}\right)=0\right)$.

Modes of jumping. We observed several cases in which a leg quickly sank under the water surface after the distal end of the leg pierced the meniscus during the stroke at the average depth of $3.7 \mathrm{~mm}$ (Fig. 3c), which is close to the sinking depth of a long thin rigid cylinder $\sqrt{2} l_{c}(3.8 \mathrm{~mm}$ for water) in a quasi-static condition $^{14,15}$ (Supplementary Fig. 4 and Supplementary Note 4). For a relatively long maximum downward reach of legs $(L>\sqrt{2})$, the excessive angular leg velocity leads to the dimple depth deeper than the sinking depth $\sqrt{2} l_{c}$ and penetration of water surface; this mode of jump is referred to as the meniscus breaking jump. The jumps that do not involve meniscus breaking can be theoretically categorized into two types: pre-takeoff closing and post-takeoff closing jumps, depending on when the legs are fully closed. In the pre-takeoff closing jumps, the legs complete their rotation before leaving the water surface $\left(\omega t_{c}=\pi / 2\right.$ and $\left.H\left(t_{c}\right)>0\right)$, whereas in the post-takeoff closing jumps, the legs are fully rotated in the air after 
takeoff $\left(\omega t_{t}<\pi / 2\right.$ and $\left.H\left(t_{t}\right)=0\right)$. Here, $t_{c}$ indicates the instant of the end of leg closing movements. Only two of these modes of jumping, the post-takeoff closing and meniscus breaking jumps (surface breaking by one or two legs) were observed in water striders as described in Fig. 3. This classification is important for model predictions (Fig. 4; see below).

The optimal jump and test of the model predictions. When we solved equation (3) and used the parameters extracted directly from the videos of jumps of the actual individual water striders, the model reasonably predicted the observed maximum dimple depth (Supplementary Note 5 and Supplementary Fig. 5a). When we used the parameters extracted from the videos to calculate the takeoff velocity of a water strider (via integrating the instantaneous net force on the body over time until the tip of leg reached the zero depth position, $t=t_{t}$ ), the results reasonably agreed with the empirically measured takeoff velocity (Supplementary Note 6 and Supplementary Fig. 5b,c). These calculations indicated that the model correctly approximates the physical processes involved in jumping.

Using the model, we derived the theoretical predictions about the optimal vertical jumping behaviour assuming that predatormediated natural selection maximizes the vertical takeoff speed. Fast vertical takeoff is important for survival because it quickly removes the insect from the vicinity of the approaching predators such as fish and backswimmers, which attack upwards from under the water surface ${ }^{6,7}$. We compared the theoretical predictions with empirical data from slow motion videos of five species of water striders with different body size and leg morphology: G. remigis, G. comatus, G. latiabdominis, G. gracilicornis and A. paludum (body mass and leg morphology are described in Supplementary Table 1; see Methods section for details). a

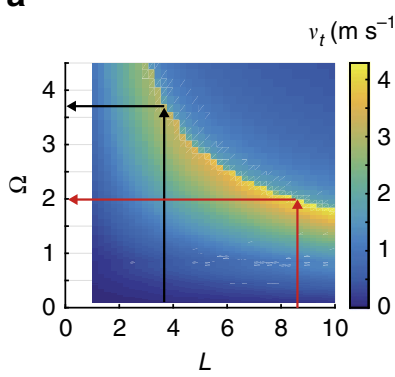

d

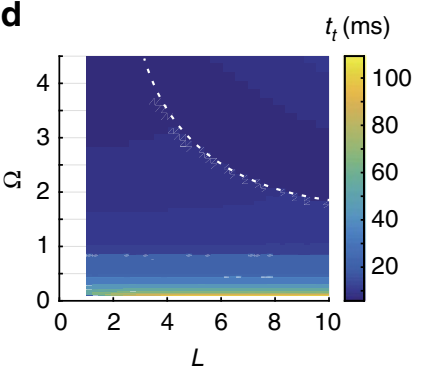

b

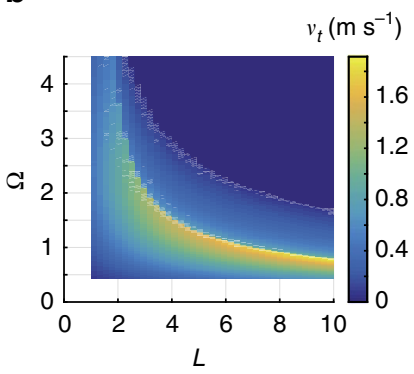

e
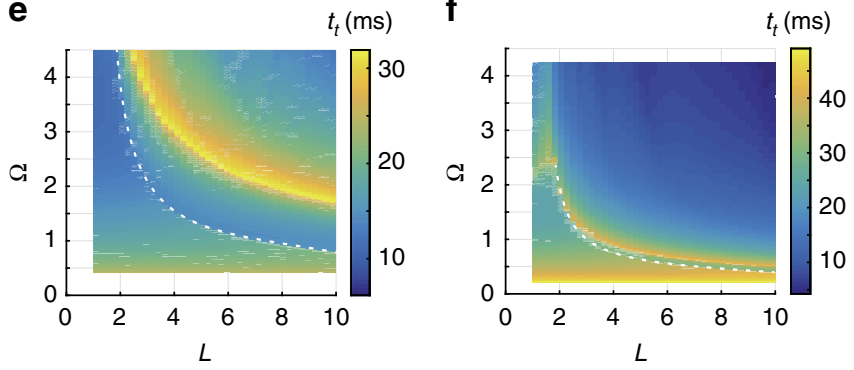

f

C

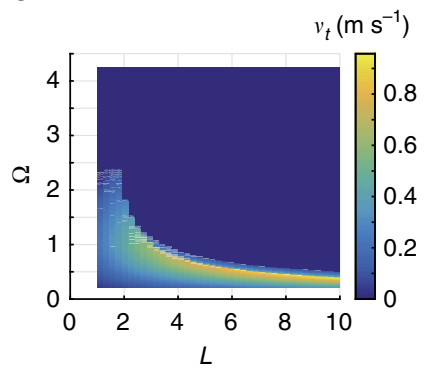

30

20 10

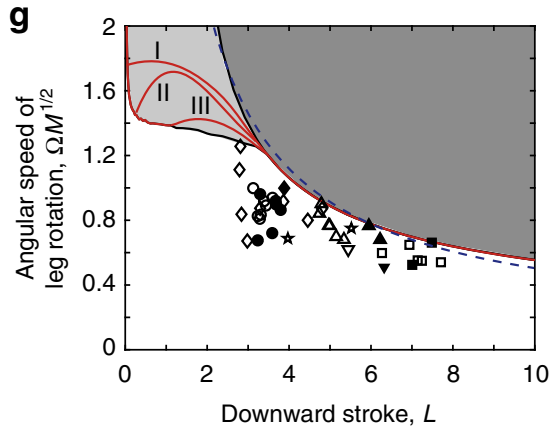


First, we calculated the predicted takeoff speed as a function of three variables that can be derived from empirical measurements on insects: the dimensionless angular velocity of the leg $\Omega$ (which is directly related to the angular velocity of leg rotation $\omega$, which in turn can be calculated from empirically measured maximum downward leg velocity, $v_{s, \max }$, and the maximal reach of legs, $\Delta l$, according to the formula $\left.\omega=v_{s, \max } / \Delta l\right)$, the dimensionless maximum reach of leg $L$ (which is directly correlated with leg length), and the variable $M$ (Fig. 4), representing the body mass expressed in units of the maximal mass of water that the tibia plus tarsus can displace. Hence, the model allowed us to predict the combinations of leg morphology $L$ and behaviour $\Omega$ that result in the maximum takeoff speed (narrow yellow areas in Fig. $4 \mathrm{a}-\mathrm{c}$ ) and corresponding time to escape from water (Fig. $4 \mathrm{~d}-\mathrm{f}$ ) for insects of different sizes $M$.

For a given $M$, the range of $L$ and $\Omega$ values that maximize takeoff speed is relatively narrow (Fig. $4 \mathrm{a}-\mathrm{c}$ ). The optimal value of $\Omega$ tends to decrease with the increase of $L$. That is, if we consider an insect of a specific $M$ (that is, an insect of specific mass $m$ and tibia plus tarsus length $l_{t}$ ), we expect that the longer is the femur (resulting in longer $L$ ), the slower should be the leg movements to produce the optimal jump (slower $\Omega$ ). For insects with large mass and/or short tibia plus tarsus length $l_{t}$, resulting in relatively large $M$ (example in Fig. $4 c ; M=2.0$ ), the values of $\Omega$ that may produce the maximal takeoff speed are relatively low (in Fig. 4c). However, for a typical water strider with relatively long tibia plus tarsus $l_{t}$ and small mass resulting in small $M$ (for example, $M=0.5$ in Fig. 4b), the leg rotational speeds that may produce optimal jump are relatively large (in Fig. $4 \mathrm{~b}$ ), and a water strider should be able to precisely adjust its leg rotation $\Omega$ to its leg length $L$ in order to produce the optimal jump. For example, if an insect of $M=0.1$ (Fig. 4a) has long legs $L$, then its optimal leg rotation speed $\Omega$ should be low (red arrows in Fig. 4a). But, if an insect of the same $M$ had short legs, then its optimal leg rotation would be fast (black arrows in Fig. 4a). The insects should be careful to not go over the optimal leg rotation speed because it may result in piercing of the water surface and a sudden decrease in jump performance (notice sharp transition from yellow to blue especially for large values of $L$; Fig. $4 \mathrm{a}-\mathrm{c}$ ) as the leg rotation $\Omega$ increases (also see Supplementary Fig. 5b and Supplementary Note 6). Moreover, Fig. 4d-f shows that the optimal conditions maximizing takeoff velocity also minimize the time to escape from the water surface for post-takeoff closing jump, which may increase the insects' survival rate (an alternative 3D graphical representation of Fig. 4a-f shown in Supplementary Fig. 6 and Supplementary Note 7).

Finally, to compare the theoretical predictions with corresponding empirical data from water striders of different sizes $M$, we constructed a two-dimensional regime map for the three modes of jumps in the space of two dimensionless variables derived from equation (3): the leg length $L$ and the composite variable $\Omega M^{1 / 2}$ involving leg rotation, body mass and tibia plus tarsus length (Fig. 4g). Because variation in $\Omega M^{1 / 2}$ expresses mostly the variation in $\Omega$ rather than $M$ in real striders, the variable $\Omega M^{1 / 2}$ mostly represents the behavioural trait $\Omega$, justifying our approximate view of $\Omega M^{1 / 2}$ as a behavioural index (see Supplementary Note 8 and Supplementary Fig. 7). The critical line of meniscus breaking could be simply approximated as $L \sim \Omega^{-1} M^{-1 / 2}$ (the blue dashed line in Fig. $4 \mathrm{~g}$ ) by balancing velocity of body centre $v$ of an insect and average downward velocity of the four legs with respect to the horizontal plane through body centre $v_{s}$ at $t=t_{m}$ (for the derivation, see Supplementary Note 9). The red lines in Fig. 4g, corresponding to the condition for the maximal takeoff velocity, are located in the area of pre-takeoff closing jump for insects with relatively short legs, that is, when the maximum downward reach of legs
$L \leq 3.5$. But, for long-legged insects, with $L>3.5$ typical for many water strider species, the fastest jump occurs when the insect drives its legs at the speed just below the meniscus breaking condition. We found that the jumping of water striders occurs always near the condition for the maximal takeoff velocity as shown in Fig. 4g. Most jumps occurred just a little below the critical line of meniscus breaking (Fig. $4 \mathrm{~g}$ ) as if the animals kept a certain safety margin to avoid the breaking of water surface that may dramatically decrease their chances of successful escape from predators.

\section{Discussion}

The reasonable match between the model predictions and the empirical findings for the maximal takeoff speeds suggests that water striders have the ability to adjust their behaviour (angular leg movements) to reach the optimal conditions for the fastest jump away from danger. The morphological traits appearing in our model, that is, body mass $m$, tibia plus tarsus length $l_{t}$, whole leg length $l_{l}$ and leg radius $r$, are not likely to have been optimally designed for only a single particular function, such as jump escape. However, the behavioural trait can in principle be adjusted by individuals within the animal's physical abilities. Based on our results, we hypothesize that a water strider of a given mass and morphology may control its stroke speed by modifying the leg rotation's angular velocity to attain the maximum jumping speed as an adaptation to avoid predation. This then leads to a question of whether the hypothetical optimal adjustment of the leg movements to morphology is achieved by natural selection for a 'hardwired' species-specific motor pattern or by individual learning. Our model enables the pursuit of a variety of similar questions in the future because, in principle, it may be used to predict the effect of leg angular movements as well as morphological features on jump performance.

For simplicity, we only focused on the vertical jumping with synchronized leg locomotion. In those jumps, the dimples on the water surface under mid-legs occur approximately simultaneously with the dimples under hind-legs ${ }^{11}$, justifying our simplifications for modelling purposes. Because in our model the insect body mass is located in one point of space, the 'body centre', we also ignored the distinction between downward movements of tibia and tarsus due to the actual leg being pushed away from the body centre by muscles and to the rotation of the insect's body axis during jump. The latter appears to contribute to the hind leg pushing against the water surface during the pushing stage of jump when body axis changes from horizontal to about $45^{\circ}$ by the moment of maximal dimple depth (Fig. 1b).

Despite its simplicity, our model is sufficient to provide us crucial insights into water strider's near-vertical jumping on water. The fast upward jump is the best solution to escape predators $^{6,7}$ in those situations when insects are surprised by an attack from under the water surface, while the attacker's approaching movement trajectory cannot be tracked by prey. Actually, the water striders also may move their four legs in many different ways, showing a variety of jumping trajectories and speeds, including back somersaults and an apparent ability to control their jump trajectories with respect to the direction of the approaching danger in situations when they can perceive it. The general theoretical approach in this study would be still valid for water striders' upward jumping with four legs moving differently if we build complex locomotion functions to reflect the four leg's motions of each case.

Together with Koh et al. ${ }^{11}$, our results prove that water striders are able to exploit water surface properties to optimally perform their predation avoidance jumps using elongated legs that are developmentally shaped by recently discovered genetic mecha- 
nism ${ }^{16}$, which is an apparent outcome of natural selection to avoid predator attacks. This work adds to the documented or suggested repertoire of water striders' behaviours that evolved to exploit the water surface properties: defence of territories ${ }^{17-21}$, courtship ${ }^{21-23}$, establishing dominance in agonistic interactions $^{20,24}$, defence of mates against harassment from other males during mate guarding ${ }^{25}$, sex recognition ${ }^{20,21,26}$ and sensing distribution of food in environment ${ }^{27}$ or possibly also sensing local sex ratio in a population ${ }^{28}$.

In summary, our study provides a mathematical understanding of how a biological organism may achieve the optimal level of motility on water surface by apparently tuning the behavioural trait to its morphology. Whether species-specific leg movements are innate and shaped by co-evolution with morphology, or behaviourally plastic and shaped by individual experience during jumping, remains to be determined. Many studies have not directly determined the role of individual learning in adjusting the locomotion behaviour to locomotory organs' morphology, but they generally documented a similar match between the morphology and behaviours in a variety of mostly vertebrate $\operatorname{taxa}^{29-39}$. The actual tuning by individuals of their locomotory behaviour to morphology has been documented in only some organisms. For example, juvenile Acrocephalus birds are able to learn from experience and adjust the use of perching sites and habitats in order to optimally tune the use of legs for perching to their legs' morphology ${ }^{40,41}$. It is possible that the water striders may also be able to adjust their leg movement to changes in physical conditions of jumping, highlighting the possibility that insects also are able to adjust their behaviour to morphology through individual locomotory learning.

The model opens new avenues of research. Biologists can start with our model to ask questions on the evolutionary mechanisms that shape jump-optimizing morphology and behaviour of water striders with known phylogenies ${ }^{42-44}$. Modified model can also be used to understand jumping of other insects of similar ecology, such as springtails or fishing spiders that exploit capillary force to jump from water. The fundamental concepts presented in this study can also give a guideline to develop semi-aquatic robots that aim to emulate the superior locomotory abilities of the water striders on water ${ }^{45-47}$.

\begin{abstract}
Methods
Experimental setup. For slow motion filming we used three Asian species (G. latiabdominis, G. gracilicornis and A. paludum from streams and ponds around the Seoul National University, and from ponds in Yongsan area, Seoul, Korea), and two North American species (G. remigis, G. comatus from the Huyck Preserve, NY, USA). Insects were filmed in the lab using artificial lighting (Korean species) or in the outdoors using sunlight (North American species). A water strider was induced to jump in a square acrylic bath $(70 \mathrm{~mm}$ wide) half-filled with water, and two high-speed cameras (Trouble Shooter 1000 ME; Fastec Imaging Inc., San Diego, CA, USA) were used to record the jumping behaviours at 500 or 1,000 frames per second from the front and side views simultaneously. A total of 39 jumps by 30 adult water striders and three jumps by two nymph water striders (morphology described in Supplementary Table 1) were recorded and analysed, where the inclinations of jump trajectory were between $60^{\circ}$ and $80^{\circ}$ to the horizontal with almost bilateral symmetry with respect to the leg of distortion of the water surface. Most of the jumps were stimulated by a rigid stick positioned below the water strider's body, and we only analysed the jumps verified that the insect's body or the leg was not pushed upwards by the stick. Fresh body mass of an insect was measured right after filming. Each animal was also photographed (a ruler was present in each photo to provide the scale) and the lengths of leg segments were measured from the photographs using ImageJ (http://imagej.nih.gov/ij/) $)^{48}$. The animals used in the present study were handled in accordance with institutional guidelines for the care and use of laboratory animals. Korean law does not require special permits for the use of water striders in research. The Huyck Preserve permitted us to use the water striders on their private land.
\end{abstract}

Model. Matlab was used to obtain the results described in Fig. 4, Supplementary Figs 5,6. To solve equation (3), ode15s function was used.
Code availability. The code is available from authors on request.

Data availability. All relevant data are available from authors on request.

\section{References}

1. Hu, D. L., Chan, B. \& Bush, J. W. M. The hydrodynamics of water strider locomotion. Nature 424, 663-666 (2003).

2. Gao, X. F. \& Jiang, L. Water-repellent legs of water striders. Nature 432, 36-36 (2004).

3. Hu, D. L. \& Bush, J. W. M. The hydrodynamics of water-walking arthropods. J. Fluid Mech. 644, 5-33 (2010).

4. Gao, P. \& Feng, J. J. A numerical investigation of the propulsion of water walkers. J. Fluid Mech. 668, 363-383 (2011).

5. Suter, R. et al. Locomotion on the water surface: propulsive mechanisms of the fisher spider. J. Exp. Biol. 200, 2523-2538 (1997).

6. Krupa, J. J. \& Sih, A. Comparison of antipredator responses of two related water striders to a common predator. Ethology 105, 1019-1033 (1999).

7. Haskins, K., Sih, A. \& Krupa, J. Predation risk and social interference as factors influencing habitat selection in two species of stream-dwelling waterstriders. Behav. Ecol. 8, 351 (1997).

8. Caponigro, M. A. \& Eriksen, C. H. Surface film locomotion by water strider, Gerris remigis Say. Am. Midl. Nat. 95, 268-278 (1976).

9. Li, J., Hesse, M., Ziegler, J. \& Woods, A. W. An arbitrary Lagrangian Eulerian method for moving-boundary problems and its application to jumping over water. J. Comput. Phys. 208, 289-314 (2005).

10. Burrows, M. Jumping from the surface of water by the long-legged fly Hydrophorus (Diptera, Dolichopodidae). J. Exp. Biol. 216, 1973-1981 (2013).

11. Koh, J.-S. et al. Jumping on water: surface tension-dominated jumping of water striders and robotic insects. Science 349, 517-521 (2015).

12. Lee, D.-G. \& Kim, H.-Y. The role of superhydrophobicity in the adhesion of a floating cylinder. J. Fluid Mech. 624, 23-32 (2009).

13. Vella, D. Floating objects with finite resistance to bending. Langmuir 24, 8701-8706 (2008).

14. Shi, F. et al. Towards understanding why a superhydrophobic coating is needed by water striders. Adv. Mater. 19, 2257-2261 (2007).

15. Vella, D., Lee, D. G. \& Kim, H.-Y. The load supported by small floating objects. Langmuir 22, 5979-5981 (2006).

16. Armisen, D. et al. Predator strike shapes antipredatory phenotype through new genetic interactions in water striders. Nat. Commun. 6, 8153 (2015).

17. Vepsalainen, K. \& Nummelin, M. Male territoriality in the water strider Limnioporus rufoscutellatus. Ann. Zool. Fenn. 22, 441-448 (1985).

18. Vepsalainen, K. \& Nummelin, M. Female territoriality in the water striders Gerris najas and G. cinereus. Ann. Zool. Fenn. 22, 433-439 (1985).

19. Jablonski, P. G. Intruder pressure affects territory size and foraging success in asymmetric contests in the water strider Gerris lacustris. Ethology 102, 22-31 (1996).

20. Jablonski, P. G. \& Wilcox, S. R. Signalling asymmetry in the communication of the water strider Aquarius remigis in the context of dominance and spacing in the non-mating season. Ethology 102, 353-359 (1996).

21. Wilcox, R. S. \& Spence, J. R. The mating system of two hybridizing species of water striders (Gerridae). I. Ripple signal functions. Beh. Ecol. Sociobiol. 19, 79-85 (1986).

22. Han, C. S. \& Jablonski, P. G. Female genitalia concealment promotes intimate courtship in a water strider. PLoS ONE 4, e5793 (2009).

23. Han, C. S. \& Jablonski, P. G. Male water striders attract predators to intimidate females into copulation. Nat. Commun. 1, 52 (2010).

24. Han, C. S. \& Jablonski, P. G. Role of body size in dominance interactions between male water striders, Aquarius paludum. J. Ethol. 28, 389-392 (2010).

25. Wilcox, R. S. \& Stefano, J. D. Vibratory signals enhance mate-guarding in a water strider (Hemiptera: Gerridae). J. Insect Behav. 4, 43-50 (1991).

26. Wilcox, R. S. Sex discrimination in Gerris remigis: role of surface wave signal. Science 206, 325-327 (1979).

27. Jablonski, P. G. \& Scinski, M. Water striders are prescient foragers: use of sensory information for patch assessment in food-based territoriality of Aquarius remigi (Gerridae, Heteroptera). Pol. J. Ecol. 47, 247-256 (1999).

28. Jablonski, P. G. \& Vepsalaiinen, K. Conflict between sexes in the water strider, Gerris lacustris: a test of two hypotheses for male guarding behavior. Behav. Ecol. 6, 388-392 (1995).

29. Norberg, U. M. Morphology of the wings, legs, and tail of three coniferous forest tits, the goldcrest and the tree creeper in realtionrelation to locomotor pattern and feeding station selection. Philos. Trans. R. Soc. London, B, Biol. Sci. 287, 131-165 (1979).

30. Webb, P. W. Body form, locomotion and foraging in aquatic vertebrates. Am. Zool. 24, 107-120 (1984).

31. Norberg, U. M. \& Rayner, J. M. V. Ecological morphology and flight in bats (Mammalia: Chiroptera): wing adaptations, flight performance, foraging 
strategy and echolocation. Philos. Trans. R. Soc. London, B, Biol. Sci. 316, 335-427 (1987).

32. Losos, J. B. The evolution of form and function: morphology and locomotor performance in west indian Anolis lizards. Evolution 44, 1189-1203 (1990).

33. Moreno, E. \& Carrascal, L. M. Leg morphology and feeding postures of four Parus species: an experimental ecomorphological approach. Ecology 74, 2037-2044 (1993).

34. Gerstner, C. L. Maneuverability of four species of coral-reef fish that differ in body and pectoral-fin morphology. Can. J. Zool. 77, 1102-1110 (1999).

35. Brana, F. Morphological correlates of burst speed and field movement patterns: the behavioural adjustement of locomotion in wall lizards (Podarcis muralis). Biol. J. Linn. Soc. 80, 135-146 (2003).

36. Dial, K. P. Evolution of avian locomotion: correlates of flight style, locomotor modules, nesting biology, body size, development and the origin of flapping flight. Auk 120, 941-952 (2003).

37. Stiles, F. G., Altshuler, D. L. \& Dudley, R. Wing morphology and flight behavior of some north American hummingbird species. Auk 122, 872-886 (2005).

38. Brewer, M. L. \& Hertel, F. Wing morphology and flight behavior of Pelecaniform searbirds. J. Morphol. 268, 866-877 (2007).

39. Tytell, E. D. et al. Disentangling the functional roles of morphology and motion in the swimming of fish. Integr. Comp. Biol. 50, 1140-1154 (2010).

40. Ley, H.-W. Verhaltensontogenese der Habitatwahl beim Teichrohrsanger (Acrocephalus scirpaceus). J. Ornithol. 129, 287-297 (1988).

41. Leisler, B., Ley, H. W. \& Winkler, H. Habitat, behavior and morphology of Acrocephalus warblers: an integrated analysis. Ornis Scand. 20, 181-186 (1989).

42. Damgaard, J., Andersen, N. M. \& Sperling, F. A. H. Phylogeny of the water strider genus Aquarius Schellenberg (Heteroptera: Gerridae) based on nuclear and mitochondrial DNA and morphology. Insect Syst. Evol. 31, 71-90 (2000).

43. Damgaard, J. \& Sperling, F. A. H. Phylogeny of the water strider genus Gerris Fabricius (Heteroptera: Gerridae) based on COI mtDNA, EF-1 $\alpha$ nuclear DNA and morphology. Syst. Entomol. 26, 241-254 (2001).

44. Damgaard, J. Phylogeny of the semiaquatic bugs (Hemiptera-Heteroptera, Gerromorpha). Insect Syst. Evol. 39, 431-460 (2008).

45. Zhao, J., Zhang, X. \& Pan, Q. in Proceeding of 2012 International Conference on Mechatronics and Automation (ICMA), 962-967 (IEEE, 2012).

46. Suhr, S. H., Song, Y. S., Lee, S. J. \& Sitti, M. in Proc. Robotics: Science and Systems I, 319-325 (IEEE, 2005).

47. Shin, B., Kim, H.-Y. \& Cho, K.-J. in Proceedings of the 2nd Biennial IEEE/ RAS-EMBS International Conference on Biomedical Robotics and Biomechatronics 127-131 (Scottsdale, AZ, USA, 2008).

48. Schneider, C. A., Rasband, W. S. \& Eliceiri, K. W. NIH image to ImageJ: 25 years of image analysis. Nat. Methods 9, 671-675 (2012).

\section{Acknowledgements}

This work was supported by the National Research Foundation of Korea Grants 2014023206 and NRF-2013R1A2A2A01006394, Disaster and Safety Management Insti tute Grant MPSS-CG-2016-02, and Bio-Mimetic Robot Research Center funded by Defense Acquisition Program Administration under Grant UD130070ID. The following persons helped in analysing the videos of jumping water striders: S. Song, C.K. Kang, J. Moon.

\section{Author contributions}

E.Y., S.-i.L., P.G.J. and H.-Y.K. designed the study. J.H.S., S.-i.L. and P.G.J. conducted experiments on water striders, extracted data and provided the results to E.Y., who analysed the jumping dynamics from these results and built the theoretical model. E.Y., S.-i.L., P.G.J. and H.-Y.K. discussed the results and the model, and provided feedback for model development and modifications by E.Y. E.Y. wrote the initial manuscript, which was subsequently modified, after detailed input from, and discussions with, P.G.J., S.-i.L. and H.-Y.K.

\section{Additional information}

Supplementary Information accompanies this paper at http://www.nature.com/ naturecommunications

Competing financial interests: The authors declare no competing financial interests.

Reprints and permission information is available online at http://npg.nature.com/ reprintsandpermissions/

How to cite this article: Yang, E. et al. Water striders adjust leg movement speed to optimize takeoff velocity for their morphology. Nat. Commun. 7, 13698 doi: $10.1038 /$ ncomms13698 (2016).

Publisher's note: Springer Nature remains neutral with regard to jurisdictional claims in published maps and institutional affiliations.

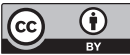

This work is licensed under a Creative Commons Attribution 4.0 International License. The images or other third party material in this article are included in the article's Creative Commons license, unless indicated otherwise in the credit line; if the material is not included under the Creative Commons license, users will need to obtain permission from the license holder to reproduce the material. To view a copy of this license, visit http://creativecommons.org/licenses/by/4.0/

(C) The Author(s) 2016 